\title{
TORIC IDEALS GENERATED BY CIRCUITS
}

\author{
JOSÉ MARTÍNEZ-BERNAL AND RAFAEL H. VILLARREAL
}

\begin{abstract}
Let $I_{\mathcal{A}}$ be the toric ideal of a homogeneous normal configuration $\mathcal{A} \subset \mathbb{Z}^{n}$. We prove that $I_{\mathcal{A}}$ is generated by circuits if and only if each unbalanced circuit of $I_{\mathcal{A}}$ has a connector which is a linear combination of circuits with a square-free term. In particular, if each circuit of $I_{\mathcal{A}}$ with non-square-free terms is balanced, then $I_{\mathcal{A}}$ is generated by circuits. As a consequence we prove that the toric ideal of a normal edge subring of a multigraph is generated by circuits with a square-free term.
\end{abstract}

\section{INTRODUCTION}

Let $\mathcal{A}$ be a finite vector configuration in $\mathbb{R}^{n}$ and let $I_{\mathcal{A}}$ be its associated toric ideal, i.e., $\mathcal{A}=\left\{v_{1}, \ldots, v_{q}\right\} \subset \mathbb{Z}^{n}$ and $I_{\mathcal{A}}$ is the prime ideal of $K[T]$ given by (see [14]):

$$
I_{\mathcal{A}}=\left(T^{a}-T^{b} \mid a=\left(a_{i}\right), b=\left(b_{i}\right) \in \mathbb{N}^{q}, \sum_{i} a_{i} v_{i}=\sum_{i} b_{i} v_{i}\right) \subset K[T],
$$

where $K[T]=K\left[T_{1}, \ldots, T_{q}\right]$ is a polynomial ring over a field $K$. Here we will use $T^{a}$ as an abbreviation for $T_{1}^{a_{1}} \cdots T_{q}^{a_{q}}$, where $a=\left(a_{i}\right)$ is a vector in $\mathbb{N}^{q}$. Let $A$ be the matrix with column vectors $v_{1}, \ldots, v_{q}$ and let $V$ be the kernel of $A$ in $\mathbb{Q}^{q}$. An integral vector $0 \neq \alpha \in V$ is called a circuit of $V$ if $\alpha$ has minimal support and its non-zero entries are relatively prime. A binomial $T^{a}-T^{b}$ is called a circuit of $I_{\mathcal{A}}$ if $a-b$ is a circuit of $V$ and $\operatorname{gcd}\left\{T^{a}, T^{b}\right\}=1$. In Section 2 the notion of a circuit is discussed in more detail. The number of circuits of $V$ is finite [16, Corollary 8.4.5] and the circuits of $V$ generate $V \cap \mathbb{Z}^{n}$ as a group [16, Theorem 8.4.10]. The notion of a circuit occurs in convex analysis [11, in the theory of toric ideals of graphs [2, 14, 15], and in matroid theory [10]. The ideal generated by the circuits of $I_{\mathcal{A}}$ has been studied in [3]. We are interested in configurations whose toric ideal $I_{\mathcal{A}}$ is generated by circuits. The best known examples of toric ideals generated by circuits come from configurations whose matrix $A$ is unimodular [14]. In Corollary 2.9 we present a larger family of toric ideals generated by circuits. Another interesting family of examples of toric ideals generated by circuits are the phylogenetic ideals studied in [5]. As noted in [9], these phylogenetic ideals actually represent the family of cut ideals of cycles. Toric ideals have been widely studied from various points of view and have interesting connections with commutative algebra, geometry, integer programming and graph theory [1, 6, 14, 16].

The configuration $\mathcal{A}$ is normal if $\mathbb{N} \mathcal{A}=\mathbb{Z} \mathcal{A} \cap \mathbb{R}_{+} \mathcal{A}$, and homogeneous if $\mathcal{A}$ lies on an affine hyperplane in $\mathbb{R}^{n}$ not containing the origin. As usual, $\mathbb{N} \mathcal{A}$ (resp. $\mathbb{Z} \mathcal{A}$ ) denotes the semigroup (resp. subgroup) of $\mathbb{Z}^{n}$ generated by $\mathcal{A}$, and $\mathbb{R}_{+} \mathcal{A}$ denotes the cone generated by $\mathcal{A}$ consisting of the linear combinations of $\mathcal{A}$ with coefficients in $\mathbb{R}_{+}=\{x \in \mathbb{R} \mid x \geq 0\}$. A binomial $T^{a}-T^{b}$ has a square-free term if $T^{a}$ is square-free or $T^{b}$ is square-free. The main result of this paper is Theorem [2.6. It shows that the toric ideal $I_{\mathcal{A}}$ of a homogeneous normal configuration is

2000 Mathematics Subject Classification. 13H10, 13B22, 13F20, 05B35.

Key words and phrases. Circuits, toric ideal, normal configuration, edge subring, multigraph.

The second author was partially supported by CONACyT grant 49251-F and SNI. 
generated by circuits if and only if every unbalanced circuit of $I_{\mathcal{A}}$ has a connector which is a $K[T]$-linear combination of circuits of $I_{\mathcal{A}}$ with a square-free term. See Definition 2.5 for a precise definition of the notion of a balanced circuit as well as for that of a connector. In particular, if $\mathcal{A}$ is homogeneous, normal and each circuit of $I_{\mathcal{A}}$ with non-square-free terms is balanced, then $I_{\mathcal{A}}$ is generated by circuits (see Corollary 2.8). As an application we prove that normal edge ideals of multigraphs are generated by circuits with a square-free term (see Theorem 3.2).

\section{Circuits of Toric ideals}

Let $\mathcal{A}=\left\{v_{1}, \ldots, v_{q}\right\} \subset \mathbb{Z}^{n}$ be a finite vector configuration, let $A$ be the integral matrix with column vectors $v_{1}, \ldots, v_{q}$, and let $I_{\mathcal{A}}$ be the associated toric ideal of $\mathcal{A}$. For $\alpha=\left(\alpha_{i}\right) \in \mathbb{R}^{q}$, its support is defined as

$$
\operatorname{supp}(\alpha)=\left\{i \mid \alpha_{i} \neq 0\right\} .
$$

Note that $\alpha=\alpha_{+}-\alpha_{-}$, where $\alpha_{+}$and $\alpha_{-}$are two non-negative vectors with disjoint support.

Definition 2.1. Let $V=\{\alpha \mid A \alpha=0\}$ be the kernel of $A$ in $\mathbb{Q}^{q}$. A circuit of $A$ is a non-zero integral vector $\alpha$ in $V$ whose support is minimal with respect to inclusion (i.e., $\operatorname{supp}(\alpha)$ does not properly contains the support of any other non-zero vector in $V$ ) and such that the non-zero entries of $\alpha$ are relatively prime. A circuit of $A$ is also called a circuit of $V$. If $\alpha$ is a circuit of $A$, we call the binomial $T^{\alpha_{+}}-T^{\alpha_{-}}$a circuit of the toric ideal $I_{\mathcal{A}}$.

Let $E=\{1, \ldots, q\}$ be the set of column labels of the matrix $A$ and let $\mathcal{I}$ be the set of subsets $B$ of $E$ for which the multiset of columns labeled by $B$ is linearly independent in $\mathbb{Q}^{n}$. Then $M[A]=(E, \mathcal{I})$ is a matroid on $E$ by [10, Proposition 1.1.1, p. 8], i.e., the collection $\mathcal{I}$ satisfies the following three conditions:

$\left(\mathrm{i}_{1}\right) \emptyset \in \mathcal{I}$.

$\left(\mathrm{i}_{2}\right)$ If $I \in \mathcal{I}$ and $I^{\prime} \subset I$, then $I^{\prime} \in \mathcal{I}$.

(i $\left.i_{3}\right)$ If $I_{1}$ and $I_{2}$ are in $\mathcal{I}$ and $\left|I_{1}\right|<\left|I_{2}\right|$, then there is an element $e$ of $I_{2} \backslash I_{1}$ such that $I_{1} \cup\{e\} \in \mathcal{I}$.

The matroid $M[A]$ is called the vector matroid of $A$ over the field $\mathbb{Q}$. The reader is referred to [10] for a general theory of matroids. The members of $\mathcal{I}$ are the independent sets of $M[A]$. A subset of $E$ that is not in $\mathcal{I}$ is called dependent. A minimal dependent set in $M[A]$ is called a circuit of $M[A]$. It is interesting to observe that there is a correspondence

$$
\begin{array}{ccc}
\text { Circuits of } A & \longrightarrow & \text { Circuits of } M[A] \\
\alpha=\left(\alpha_{1}, \ldots, \alpha_{q}\right) & \longmapsto & \operatorname{supp}(\alpha) .
\end{array}
$$

Thus the set of circuits of $A$ is an algebraic realization of the set of circuits of the vector matroid $M[A]$.

Definition 2.2. Two vectors $\alpha=\left(\alpha_{i}\right)$ and $\beta=\left(\beta_{i}\right)$ in $\mathbb{Q}^{q}$ are in harmony if $\alpha_{i} \beta_{i} \geq 0$ for all $i$.

Lemma $2.3([1])$. Let $V$ be the kernel of $A$ in $\mathbb{Q}^{q}$. If $0 \neq \alpha \in V$, then there is a circuit $\gamma \in V$ in harmony with $\alpha$ such that $\operatorname{supp}(\gamma) \subset \operatorname{supp}(\alpha)$.

A non-zero binomial $T^{a}-T^{b}$ is said to have a square-free term if $a_{i} \in\{0,1\}$ for all $i$ or $b_{i} \in\{0,1\}$ for all $i$. If $a_{i} \notin\{0,1\}$ for some $i$ and $b_{j} \notin\{0,1\}$ for some $j$, we say that the binomial $T^{a}-T^{b}$ has non-square-free terms.

Lemma 2.4. [13, Proposition 4.1] If $\mathcal{A}$ is homogeneous, normal, and $I_{\mathcal{A}}$ is minimally generated by a finite set $\mathcal{B}$ consisting of binomials, then every element of $\mathcal{B}$ has a square-free term. 
Definition 2.5. A binomial $g=T_{1}^{a_{1}} \cdots T_{q}^{a_{q}}-T_{1}^{b_{1}} \cdots T_{q}^{b_{q}}$ is called balanced if the following holds:

$$
\max \left\{a_{1}, \ldots, a_{q}\right\}=\max \left\{b_{1}, \ldots, b_{q}\right\} .
$$

If $g$ is not balanced it is called unbalanced. Let $g$ be an unbalanced binomial of the form:

$$
g=T_{1}^{b_{1}} \cdots T_{r}^{b_{r}}-T_{r+1}^{b_{r+1}} \cdots T_{s}^{b_{s}}, \quad b_{i} \geq 1 \forall i,
$$

where $1 \leq m_{1}=\max \left\{b_{1}, \ldots, b_{r}\right\}<\max \left\{b_{r+1}, \ldots, b_{s}\right\}=m_{2}$. A connector of $g$ is a binomial:

$$
T_{i_{1}} \cdots T_{i_{j}}-T_{i_{j+1}}^{c_{j+1}} \cdots T_{i_{m}}^{c_{m}}, \quad c_{i} \geq 1 \forall i
$$

with a square-free term $T_{i_{1}} \cdots T_{i_{j}}$ such that $\left\{i_{1}, \ldots, i_{j}\right\} \subset\{1, \ldots, r\}$ and the intersection of $\left\{i_{j+1}, \ldots, i_{m}\right\}$ with $\{r+1, \ldots, s\}$ is non-empty.

We come to the main result of this paper.

Theorem 2.6. Let $\mathcal{A}$ be a homogeneous normal configuration and let $I_{\mathcal{A}}$ be its toric ideal. The following are equivalent:

(a) $I_{\mathcal{A}}$ is generated by a finite set of circuits.

(b) $I_{\mathcal{A}}$ is generated by a finite set of circuits with a square-free term.

(c) Every unbalanced circuit of $I_{\mathcal{A}}$ has a connector which is a linear combination (with coefficients in $K[T]$ ) of circuits of $I_{\mathcal{A}}$ with a square-free term.

Proof. Let $R=k\left[x_{1}^{ \pm 1}, \ldots, x_{n}^{ \pm 1}\right]$ be the ring of Laurent polynomials over a field $K$ and let $K[F]$ be the subring of $R$ generated by $F=\left\{x^{v_{1}}, \ldots, x^{v_{q}}\right\}$. Since $\mathcal{A}$ is normal, we get that $K[F]$ is normal, i.e., $\overline{K[F]}=K[F]$, where $\overline{K[F]}$ is the integral closure of $K[F]$ in its field of fractions. As $\mathcal{A}$ is homogeneous, we get that any binomial $T^{a}-T^{b}$ in $I_{\mathcal{A}}$ is homogeneous with respect to the standard grading of $K[T]=K\left[T_{1}, \ldots, T_{q}\right]$ induced by setting $\operatorname{deg}\left(T_{i}\right)=1$ for all $i$, a fact that will be used repeatedly below without any further notice.

(a) $\Rightarrow\left(\right.$ b): The toric ideal $I_{\mathcal{A}}$ is minimally generated by a finite set $\mathcal{B}$ of circuits. Thus, by Lemma 2.4, each binomial of $\mathcal{B}$ is a circuit with a square-free term.

$(b) \Rightarrow(c)$ : Let $g$ be an unbalanced circuit of $A$ :

$$
g=T_{1}^{b_{1}} \cdots T_{r}^{b_{r}}-T_{r+1}^{b_{r+1}} \cdots T_{s}^{b_{s}}, \quad b_{i} \geq 1 \forall i,
$$

where $1 \leq m_{1}=\max \left\{b_{1}, \ldots, b_{r}\right\}<\max \left\{b_{r+1}, \ldots, b_{s}\right\}=m_{2}$. We may assume $m_{2}=b_{r+1}$. Then

$$
\left(x^{v_{1}} \cdots x^{v_{r}} / x^{v_{r+1}}\right)^{m_{1}} \in K[F] .
$$

The element $x^{v_{1}} \cdots x^{v_{r}} / x^{v_{r+1}}$ is in the field of fractions of $K[F]$ and by Eq. (2.1) it is integral over $K[F]$. Hence, by the normality of $K[F]$, the element $x^{v_{1}} \cdots x^{v_{r}} / x^{v_{r+1}}$ is in $K[F]$. Since $K[F]$ is generated as a $K$-vector space by Laurent monomials of the form $x^{a}$, with $a \in \mathbb{N} \mathcal{A}$, it is not hard to see that there is a monomial $T^{\gamma}$ such that

$$
T_{1} \cdots T_{r}-T_{r+1} T^{\gamma} \in I_{\mathcal{A}} .
$$

This is a connector of $g$ and by hypothesis it is a linear combination of circuits of $I_{\mathcal{A}}$ with a square-free term.

(c) $\Rightarrow$ (a): By Lemma 2.4, the toric ideal $I_{\mathcal{A}}$ is minimally generated by a finite set

$$
\mathcal{B}=\left\{f_{1}, \ldots, f_{m}\right\}
$$

consisting of binomials with a square-free term. We will show, by induction on the degree, that each one of the $f_{i}$ 's is a linear combination of circuits. The degree is taken with respect to the standard grading of $K[T]$. 
Let $f$ be a binomial in $\mathcal{B}$. We may assume that $f$ has the form:

$$
f=T_{1} \cdots T_{p}-T_{p+1}^{a_{p+1}} \cdots T_{\ell}^{a_{\ell}}, \quad a_{i} \geq 1 \forall i, \ell \leq q .
$$

Assume that $f$ is not a circuit. Then by Lemma 2.3 there is a circuit in $I_{\mathcal{A}}$ (permuting variables if necessary) of the form

$$
g=T_{1}^{b_{1}} \cdots T_{r}^{b_{r}}-T_{p+1}^{b_{p+1}} \cdots T_{s}^{b_{s}}, \quad b_{i} \geq 1 \forall i,
$$

with $r<p$ or $s<\ell$. We set $m_{1}=\max \left\{b_{1}, \ldots, b_{r}\right\}$ and $m_{2}=\max \left\{b_{p+1}, \ldots, b_{s}\right\}$.

We claim that there exist binomials $h$ and $h_{1}$ (we allow $h=h_{1}$ or $h=0$ ) in $I_{\mathcal{A}}$ of degree less than $\operatorname{deg}(f)=p$ and a binomial $h_{2}$ which is a linear combination of circuits of $I_{\mathcal{A}}$ such that $f$ is in the ideal of $K[T]$ generated by $g, h, h_{1}, h_{2}$. To prove this we consider the following two cases.

Case (A): $r=p$ and $s<\ell$. Then

$$
g=T_{1}^{b_{1}} \cdots T_{p}^{b_{p}}-T_{p+1}^{b_{p+1}} \cdots T_{s}^{b_{s}} .
$$

Subcase $\left(\mathrm{A}_{1}\right): b_{i}=1$ for $i=1, \ldots, p$. Then we can write

$$
f-g=T_{p+1}^{b_{p+1}} \cdots T_{s}^{b_{s}}-T_{p+1}^{a_{p+1}} \cdots T_{\ell}^{a_{\ell}}=T_{p+1} h,
$$

for some binomial $0 \neq h \in I_{\mathcal{A}}$ (recall that $I_{\mathcal{A}}$ is a prime ideal) with $\operatorname{deg}(h)<\operatorname{deg}(f)=p$.

Subcase $\left(\mathrm{A}_{2}\right): b_{i}=1$ for $i=p+1, \ldots, s$. Then

$$
g=T_{1}^{b_{1}} \cdots T_{p}^{b_{p}}-T_{p+1} \cdots T_{s} .
$$

By subcase $\left(\mathrm{A}_{1}\right)$, we may assume that $b_{i} \geq 2$ for some $1 \leq i \leq p$. Then, on the one hand, by the homogeneity of $g, p+1 \leq \sum_{i=1}^{p} b_{i}=s-p$, so $2 p+1 \leq s$. On the other hand, by the homogeneity of $f, p \geq \ell-p \geq s-p+1$, so $2 p-1 \geq s$. This is a contradiction. So this case cannot occur.

Subcase $\left(\mathrm{A}_{3}\right): b_{i} \geq 2$ for some $1 \leq i \leq p, b_{p+j} \geq 2$ for some $1 \leq j \leq s-p$, and $m_{1} \geq m_{2}$. Then

$$
\begin{gathered}
f=T_{1} \cdots T_{p}-T_{p+1}^{a_{p+1}} \cdots T_{\ell}^{a_{\ell}}, \\
g=T_{1}^{b_{1}} \cdots T_{p}^{b_{p}}-T_{p+1}^{b_{p+1}} \cdots T_{s}^{b_{s}} .
\end{gathered}
$$

For simplicity of notation we may assume that $m_{1}=b_{1}$. Using that $g \in I_{\mathcal{A}}$ and $m_{1} \geq m_{2} \geq 2$, we get

$$
\left(x^{v_{p+1}} \cdots x^{v_{s}} / x^{v_{1}}\right)^{m_{2}} \in K[F] .
$$

Hence, by the normality of $K[F]$, there is a monomial $T^{\gamma}$ such that the binomial

$$
h_{1}=T_{p+1} \cdots T_{s}-T_{1} T^{\gamma}
$$

is in $I_{\mathcal{A}}$. The binomial $h_{1}$ is non-zero and has degree less than $\operatorname{deg}(f)$ because $s-p<\ell-p \leq p$; the second inequality follows from the homogeneity of $f$. Let

$$
T^{\delta}=T_{p+1}^{a_{p+1}} \cdots T_{\ell}^{a_{\ell}} / T_{p+1} \cdots T_{s} .
$$

We have

$$
f+h_{1} T^{\delta}=f+\left(T_{p+1} \cdots T_{s}-T_{1} T^{\gamma}\right) T^{\delta}=T_{1} \cdots T_{p}-T_{1} T^{\gamma} T^{\delta}=T_{1} h,
$$

where $0 \neq h \in I_{\mathcal{A}}$ and $\operatorname{deg}(h)<p$.

Subcase $\left(\mathrm{A}_{4}\right): b_{i} \geq 2$ for some $1 \leq i \leq p, b_{p+j} \geq 2$ for some $1 \leq j \leq s-p$, and $m_{1}<m_{2}$. Since $g$ is an unbalanced circuit, by hypothesis $g$ has a connector

$$
h_{2}=T_{i_{1}} \cdots T_{i_{k}}-T_{i_{k+1}} T^{\gamma}, \quad i_{1}<\cdots<i_{k}
$$


TORIC IDEALS GENERATED BY CIRCUITS

with $i_{k+1} \in\{p+1, \ldots, s\},\left\{i_{1}, \ldots, i_{k}\right\} \subset\{1, \ldots, p\}$ and such that $h_{2}$ is a linear combination of circuits of $I_{\mathcal{A}}$. Set

$$
T^{\delta}=T_{1} \cdots T_{p} / T_{i_{1}} \cdots T_{i_{k}}
$$

If $f=h_{2} T^{\delta}$, then $T^{\delta}=1$ and $f=h_{2}$. If $f \neq h_{2} T^{\delta}$, then we can write

$$
f-h_{2} T^{\delta}=T_{i_{k+1}} T^{\gamma} T^{\delta}-T_{p+1}^{a_{p+1}} \cdots T_{\ell}^{a_{\ell}}=T_{i_{k+1}} h,
$$

with $0 \neq h \in I_{\mathcal{A}}$ and $\operatorname{deg}(h)<p$.

Case (B): $r<p$ and $s \leq \ell$. In this case

$$
\begin{gathered}
f=T_{1} \cdots T_{p}-T_{p+1}^{a_{p+1}} \cdots T_{\ell}^{a_{\ell}}, \\
g=T_{1}^{b_{1}} \cdots T_{r}^{b_{r}}-T_{p+1}^{b_{p+1}} \cdots T_{s}^{b_{s}} .
\end{gathered}
$$

Subcase $\left(\mathrm{B}_{1}\right): b_{i}=1$ for $i=1, \ldots, r$. Then

$$
f-g T_{r+1} \cdots T_{p}=T_{p+1}^{b_{p+1}} \cdots T_{s}^{b_{s}} T_{r+1} \cdots T_{p}-T_{p+1}^{a_{p+1}} \cdots T_{\ell}^{a_{\ell}}=T_{p+1} h,
$$

where $0 \neq h \in I_{\mathcal{A}}$ and $\operatorname{deg}(h)<p$.

Subcase $\left(\mathrm{B}_{2}\right): b_{i}=1$ for $i=p+1, \ldots, s$. Let

$$
T^{\gamma}=T_{p+1}^{a_{p+1}} \cdots T_{\ell}^{a_{\ell}} / T_{p+1} \cdots T_{s}
$$

Then we have

$$
f-g T^{\gamma}=T_{1} \cdots T_{p}-T_{1}^{b_{1}} \cdots T_{r}^{b_{r}} T^{\gamma}=T_{1} h
$$

where $0 \neq h \in I_{\mathcal{A}}$ and $\operatorname{deg}(h)<p$.

Subcase $\left(\mathrm{B}_{3}\right): b_{i} \geq 2$ for some $1 \leq i \leq r, b_{j} \geq 2$ for some $p+1 \leq j \leq s$, and $m_{1} \leq m_{2}$. We may assume $m_{2}=b_{p+1}$. Using that $g \in I_{\mathcal{A}}$ and $m_{2} \geq m_{1} \geq 2$, we get

$$
\left(x^{v_{1}} \cdots x^{v_{r}} / x^{v_{p+1}}\right)^{m_{1}} \in K[F] .
$$

Hence, by the normality of $K[F]$, there is a monomial $T^{\gamma}$ such that the binomial

$$
h_{1}=T_{1} \cdots T_{r}-T_{p+1} T^{\gamma}
$$

is in $I_{\mathcal{A}}$. The binomial $h_{1}$ is non-zero and has degree less than $\operatorname{deg}(f)$ because $r<p$. Then we have

$$
\begin{aligned}
f-h_{1} T_{r+1} \cdots T_{p} & =f-\left(T_{1} \cdots T_{r}-T_{p+1} T^{\gamma}\right) T_{r+1} \cdots T_{p} \\
& =T_{p+1} T^{\gamma} T_{r+1} \cdots T_{p}-T_{p+1}^{a_{p+1}} \cdots T_{\ell}^{a_{\ell}}=T_{p+1} h,
\end{aligned}
$$

where $0 \neq h \in I_{\mathcal{A}}$ and $\operatorname{deg}(h)<p$.

$\underline{\text { Subcase }}\left(\mathrm{B}_{4}\right): b_{i} \geq 2$ for some $1 \leq i \leq r, b_{j} \geq 2$ for some $p+1 \leq j \leq s$, and $m_{1}>m_{2}$. Since $g$ is an unbalanced circuit, by hypothesis $g$ has a connector

$$
h_{2}=T_{i_{k+1}} \cdots T_{i_{k+t}}-T_{i_{d}} T^{\gamma}, \quad i_{k+1}<\cdots<i_{k+t},
$$

with $\left\{i_{k+1}, \cdots, i_{k+t}\right\} \subset\{p+1, \ldots, s\}, i_{d} \in\{1, \ldots, r\}$, and such that $h_{2}$ is a linear combination of circuits of $I_{\mathcal{A}}$. Set

$$
T^{\delta}=T_{p+1}^{a_{p+1}} \cdots T_{\ell}^{a_{\ell}} / T_{i_{k+1}} \cdots T_{i_{k+t}} .
$$

If $f=-h_{2} T^{\delta}$, then $T^{\delta}=1$ and $f=-h_{2}$. If $f \neq-h_{2} T^{\delta}$, then we can write

$$
f+h_{2} T^{\delta}=T_{1} \cdots T_{p}-T_{i_{d}} T^{\gamma} T^{\delta}=T_{i_{d}} h,
$$

with $0 \neq h \in I_{\mathcal{A}}$ and $\operatorname{deg}(h)<p$. This completes the proof of the claim. 
We are now ready to show that each $f_{i}$ in $\mathcal{B}$ is a linear combination of circuits. We proceed by induction on $\operatorname{deg}\left(f_{i}\right)$. Let $p=\min \left\{\operatorname{deg}\left(f_{i}\right) \mid 1 \leq i \leq m\right\}$ be the initial degree of $I_{\mathcal{A}}$. If $f_{i}$ is a binomial in $\mathcal{B}$ of degree $p$, then either $f_{i}$ is a circuit or $f_{i}$ is not a circuit and by the claim $f_{i}$ is a linear combination of circuits (notice that in this case $h=h_{1}=0$ because there are no non-zero binomials in $I_{\mathcal{A}}$ of degree less than $p$ ). Let $d$ be an integer greater than $p$ and let $f_{k}$ be a binomial of $\mathcal{B}$ of degree $d$ (if any). Assume that each $f_{i}$ of degree less than $d$ is a linear combination of circuits. If $f_{k}$ is a circuit there is nothing to prove. If $f_{k}$ is not a circuit, then by the claim (or more precisely by Eqs. (2.2)-(2.8)) we can write

$$
f_{k}=\lambda g+\mu h+\mu_{1} h_{1}+\mu_{2} h_{2},
$$

where $\lambda, \mu, \mu_{1}, \mu_{2}$ are monomials, $h, h_{1}$ are binomials in $I_{\mathcal{A}}$ of degree less than $d=\operatorname{deg}\left(f_{k}\right), h_{2}$ is a linear combination of circuits, and $g$ is a circuit. Since $I_{\mathcal{A}}$ is a graded ideal with respect to the standard grading of $K\left[T_{1}, \ldots, T_{q}\right]$, we get that $h$ and $h_{1}$ are linear combinations of binomials in $\mathcal{B}$ of degree less than $d$. Therefore by Eq. (2.9) and the induction hypothesis, we conclude that $f_{k}$ is a linear combination of circuits. Therefore the ideal $I_{\mathcal{A}}$ is generated by a finite set of circuits.

Remark 2.7. In the proof of Theorem 2.6 (from (c) to (a)), the subcases $\left(A_{3}\right),\left(B_{1}\right)$, and $\left(B_{3}\right)$ cannot occur. Indeed, since $f_{i}$ belongs to a minimal system of binomial generators of the toric ideal it cannot be written as a linear combination of binomials of the toric ideal of degree strictly smaller. In Eq. (2.9) either $\lambda$ or $\mu_{2}$ has to be nonzero.

The following result will be used in Section 3 to show a class of toric ideals generated by circuits.

Corollary 2.8. Let $\mathcal{A}$ be a homogeneous normal configuration and let $I_{\mathcal{A}}$ be its toric ideal. If each circuit of $I_{\mathcal{A}}$ with non-square-free terms is balanced, then $I_{\mathcal{A}}$ is generated by a finite set of circuits with a square-free term.

Proof. The circuits of $I_{\mathcal{A}}$ satisfy condition (c) of Theorem 2.6. Indeed, let $f$ be an unbalanced circuit of $I_{\mathcal{A}}$. Then $f$ has a square-free term by hypothesis. Thus $f$ is a circuit with a square-free term and it is a connector of $f$. Hence the result follows from Theorem 2.6.

Corollary 2.9. Let $\mathcal{A} \subset \mathbb{N}^{n} \backslash\{0\}$ be a homogeneous configuration and let $I_{\mathcal{A}}$ be its toric ideal. If each circuit of $I_{\mathcal{A}}$ has a square-free term, then $\mathcal{A}$ is normal and $I_{\mathcal{A}}$ is generated by a finite set of circuits with a square-free term.

Proof. The normality of $\mathcal{A}$ follows from [4, Theorem 2.3]. Since the circuits of $I_{\mathcal{A}}$ satisfy condition (c) of Theorem 2.6, we get that $I_{\mathcal{A}}$ is generated by a finite set of circuits with a square-free term.

\section{TORIC IDEALS OF NORMAL EDGE SUBRINGS}

Let $G$ a multigraph with vertex set $X=\left\{x_{1}, \ldots, x_{n}\right\}$, i.e., $G$ is obtained from a simple graph by allowing multiple edges and multiple loops. Thus the edges of $G$ have the form $\left\{x_{i}, x_{j}\right\}$. If $e=\left\{x_{i}, x_{j}\right\}$ is an edge of $G$, its characteristic vector is given by $v_{e}=e_{i}+e_{j}$, where $e_{i}$ is the $i$ th unit vector in $\mathbb{R}^{n}$. Notice that if $e$ is a loop, i.e., if $i=j$, then $v_{e}=2 e_{i}$. The incidence matrix of $G$, denoted by $A$, is the matrix whose column vectors are the characteristic vectors of the edges and loops of $G$. Since we are allowing multiple edges some of the columns of $A$ may be repeated. Let $v_{1}, \ldots, v_{q}$ be the characteristic vectors of the edges and loops of $G$ and let $\mathcal{A}=\left\{v_{1}, \ldots, v_{q}\right\}$ be its associated vector configuration. 
TORIC IDEALS GENERATED BY CIRCUITS

Let $K\left[T_{1}, \ldots, T_{q}\right]$ be a polynomial ring over a field $K$. The edge subring of $G$ is the monomial subring:

$$
K[G]=K\left[x^{v_{1}}, \ldots, x^{v_{q}}\right] \subset K\left[x_{1}, \ldots, x_{n}\right],
$$

where $K\left[x_{1}, \ldots, x_{n}\right]$ is a polynomial ring with coefficients in $K$. It is well known that the toric ideal $I_{\mathcal{A}}$ is the kernel of the following epimorphism of $K$-algebras

$$
K\left[T_{1}, \ldots, T_{q}\right] \longrightarrow K[G]
$$

induced by $T_{i} \mapsto x^{v_{i}}$.

Proposition 3.1. If $f=T^{a}-T^{b}$ is a circuit of the toric ideal $I_{\mathcal{A}}$, then $f$ has a square-free term or $f$ has non-square-free terms and $\max _{i}\left\{a_{i}\right\}=\max _{i}\left\{b_{i}\right\}=2$.

Proof. If $G$ is a simple graph, the result was shown in [15, Corollary 4.1]. The general case, i.e., the multigraph case, follows using an identical argument as the one given in [15].

We come to the main application of this paper.

Theorem 3.2. Let $G$ be a multigraph and let $I_{\mathcal{A}}$ be the toric ideal of the edge subring $K[G]$. Then $K[G]$ is normal if and only if $I_{\mathcal{A}}$ is generated by circuits with a square-free term.

Proof. $\Rightarrow$ ) This direction follows at once applying Proposition 3.1 and Corollary 2.8.

$\Leftarrow)$ It is seen using a description of the integral closure of the edge subring $K[G]$ given in [12 (cf. [2, Proposition 5.9]).

Definition 3.3. A sub-multigraph $H$ of $G$ is called a circuit of $G$ if $H$ has one of the following forms:

(a) $H$ is an even cycle.

(b) $H$ consists of two odd cycles intersecting in exactly one vertex; a loop is regarded as an odd cycle of length 1 .

(c) $H$ consists of two vertex disjoint odd cycles joined by a path.

The circuits of $G$ are in one to one correspondence with the circuits of $I_{\mathcal{A}}$ as we now explain, see [15] for a detailed discussion. Any circuit $H$ of $G$ can be regarded as an even closed walk

$$
w=\left\{w_{0}, w_{1}, \ldots, w_{r}, w_{0}\right\}
$$

where $r$ is even, $w_{0}, w_{1}, \ldots, w_{r}$ are the vertices of $H$ (we allow repetitions) and $\left\{w_{i}, w_{i+1}\right\}$ is an edge or loop of $G$ for all $i$. Then the binomial $T_{w}=T_{1} T_{3} \cdots T_{r-1}-T_{2} T_{4} \cdots T_{r}$ is in $I_{\mathcal{A}}$, where $f_{i}=w_{i-1} w_{i}$ and $T_{i}$ maps to $f_{i}$ for all $i$.

Remark 3.4. The circuits of $I_{\mathcal{A}}$ with a square-free term correspond to the following types of circuits of $G$ :

(a) Even cycles.

(b) Two odd cycles intersecting in exactly one vertex.

(c) Two vertex disjoint odd cycles joined by an edge.

Thus if $K[G]$ is a normal subring, by Theorem 3.2 we obtain a very precise graph theoretical description of a generating set of circuits for $I_{\mathcal{A}}$.

Toric ideals of edge subrings of oriented graphs were studied in [7, 8]. In this case the toric ideal is also generated by circuits and the circuits correspond to the cycles of the graph. 
Acknowledgments. We thank the referees for a careful reading of the paper and for the improvements suggested.

Note added in proof: Apostolos Thoma has pointed out to us that the condition of Theorem 3.2 is not sufficient for the normality of the edge subring. A sufficient condition is the one given in Corollary 2.9.

\section{REFERENCES}

[1] M. Barile, M. Morales, and A. Thoma, Set-theoretic complete intersections on binomials, Proc. Amer. Math. Soc. 130 (2002), 1893-1903.

[2] I. Bermejo, P. Gimenez and A. Simis, Polar syzigies in characteristic zero: the monomial case, J. Pure Appl. Algebra 213 (2009), no. 1, 1-21.

[3] T. Bogart, A. N. Jensen and R. R. Thomas, The circuit ideal of a vector configuration, J. Algebra 309 (2007), no. 2, 518-542.

[4] V. Bonanzinga, C. Escobar and R. H. Villarreal, On the normality of Rees algebras associated to totally unimodular matrices, Results Math. 41, 3/4, (2002), 258-264.

[5] J. Chifman and S. Petrović, Toric ideals of phylogenetic invariants for the general group-based model on claw trees $K_{1, n}$, Proceedings of the Second international conference on Algebraic Biology, to appear. (2007), Preprint. arXiv:0702368v3.

[6] W. Fulton, Introduction to Toric Varieties, Princeton University Press, 1993.

[7] I. Gitler, E. Reyes and R. H. Villarreal, Ring graphs and toric ideals, Electronic Notes in Discrete Mathematics 28C (2007), 393-400.

[8] I. Gitler, E. Reyes and R. H. Villarreal, Ring graphs and complete intersection toric ideals, Discrete Math., to appear.

[9] U. Nagel and S. Petrović, Properties of cut ideals associated to ring graphs, J. Commut. Algebra, to appear. (2008), Preprint. arXiv:0806.0585 2.

[10] J. Oxley, Matroid Theory, Oxford University Press, Oxford, 1992.

[11] R. T. Rockafellar, The elementary vectors of a subspace of $R^{N}$, in Combinatorial Mathematics and its Applications, Proc. Chapel Hill Conf., Univ. North Carolina Press, 1969, pp. 104-127.

[12] A. Simis, W. V. Vasconcelos and R. H. Villarreal, The integral closure of subrings associated to graphs, J. Algebra 199 (1998), 281-289.

[13] A. Simis and R. H. Villarreal, Constraints for the normality of monomial subrings and birationality, Proc. Amer. Math. Soc. 131 (2003), 2043-2048.

[14] B. Sturmfels, Gröbner Bases and Convex Polytopes, University Lecture Series 8, American Mathematical Society, Rhode Island, 1996.

[15] R. H. Villarreal, Rees algebras of edge ideals, Comm. Algebra 23 (1995), 3513-3524.

[16] R. H. Villarreal, Monomial Algebras, Monographs and Textbooks in Pure and Applied Mathematics 238, Marcel Dekker, Inc., New York, 2001.

Departamento de Matemáticas, Centro de Investigación y de Estudios Avanzados del IPN, Apartado Postal 14-740, 07000 Mexico City, D.F.

E-mail address: jmb@math.cinvestav.mx

Departamento de Matemáticas, Centro de Investigación y de Estudios Avanzados del IPN, Apartado Postal 14-740, 07000 Mexico City, D.F.

E-mail address: vila@math.cinvestav.mx

$U R L$ : http://www. math. cinvestav.mx/ vila/ 